ELECTRONIC RESEARCH ANNOUNCEMENTS OF THE AMERICAN MATHEMATICAL SOCIETY

Volume 3, Pages $72-77$ (August 19, 1997)

S $1079-6762(97) 00027-9$

\title{
PERTURBATION EXPANSIONS FOR EIGENVALUES AND EIGENVECTORS FOR A RECTANGULAR MEMBRANE SUBJECT TO A RESTORATIVE FORCE
}

\author{
JOYCE R. MCLAUGHLIN AND ARTURO PORTNOY
}

(Communicated by Michael Taylor)

\begin{abstract}
Series expansions are obtained for a rich subset of eigenvalues and eigenfunctions of an operator that arises in the study of rectangular membranes: the operator is the 2-D Laplacian with restorative force term and Dirichlet boundary conditions. Expansions are extracted by considering the restorative force term as a linear perturbation of the Laplacian; errors of truncation for these expansions are estimated. The criteria defining the subset of eigenvalues and eigenfunctions that can be studied depend only on the size and linearity of the perturbation. The results are valid for almost all rectangular domains.
\end{abstract}

\section{INTRODUCTION}

In this summary we consider a rectangular, homogeneous membrane and a force acting on it that depends linearly on the displacement from equilibrium. The eigenvalues and eigenfunctions are well known when the restorative force term is zero, prompting us to treat the case of nontrivial restorative force as a linear perturbation of the homogeneous case.

The distance between unperturbed eigenvalues is very important in studying how perturbed eigenvalues and eigenfunctions behave. It turns out that even in the cases where all unperturbed eigenvalues are distinct, sufficiently large eigenvalues can get arbitrarily close together. These arbitrarily small differences appear as divisors in the expansions, making it difficult to prove the expansions worthy.

This paper is inspired by the work of McLaughlin and Hald, [MH] and [HM]. They derive asymptotic expansions for almost all eigenvalue, eigenfunction pairs in order to solve the inverse nodal problem, with corresponding error estimates given in several norms. They estimate very sharp error bounds for specific truncations of the perturbation expansion and show that their expansions hold for almost all eigenvalues and eigenfunctions. The criteria defining this set of "good" eigenvalue, eigenfunction pairs are not completely known a priori. They present three a priori conditions and a fourth condition that depends on the values of the perturbed eigenvalues. We present the form and error bounds of arbitrary truncations, and

Received by the editors May 16, 1997.

1991 Mathematics Subject Classification. Primary 35P20.

Key words and phrases. Perturbation expansion, eigenvalue, eigenvector, membrane, inverse nodal problem. 
we do that for a well-defined (defined a priori) subset consisting of almost all eigenvalues. Also, our restrictions on the parameters, including the smoothness of $q$, are less stringent. We estimate cruder bounds on the error of truncation. We do not give details here, but in $[\mathrm{MP}]$ we show that if the additional restrictions on smoothness and other parameters that are imposed in $[\mathrm{HM}]$ are made, then their sharper estimates are valid for our collection of "good" eigenvalue, eigenfunction pairs. Here we provide a rich subset of eigenvalue, eigenfunction pairs, determined exclusively with a priori lattice point requirements, that can be used to obtain our error bounds, to obtain the error bounds in $[\mathrm{HM}]$ and to solve the inverse nodal problem defined in $[\mathrm{HM}]$.

The strategy used to find these expansions follows:

- We build on three key results from [HM]: The first tells us that for almost all rectangular membranes, almost all eigenvalues are well separated; the second tells us that eigenvalues with close neighbors rarely have corresponding eigenfunctions with similar oscillation properties; finally, the third condition eliminates points in the lattice that are too close to either axis. All three results define "thin" subsets of "bad" lattice points with a priori criteria. Disregarding these subsets is important but insufficient to justify the expansions we give here.

- We add an additional a priori condition which strips another thin subset of "bad" eigenvalues, allowing us to prove the expansions convergent for the remaining large set of eigenvalues and eigenfunctions. Geometric considerations are used to prove that this new set is "thin". This condition is the main effort of this work. It replaces the fourth criterion imposed in $[\mathrm{HM}]$, p. 68.

- Explicit $L^{\infty}$ bounds are given for the errors of truncation of the eigenfunction and eigenvalue expansions.

Four other papers are listed in the Bibliography that deal with the solution of problems similar to ours: $[\mathrm{FKT}],[\mathrm{F}],[\mathrm{KP}]$ and $[\mathrm{KP} 2]$. We point out differences, similarities, advantages and disadvantages between our approach and theirs in what follows.

First, we should define the problem these three papers examine: the differential operator is the same, but the boundary conditions are different. All three consider an unbounded domain, but assume $q$ to be periodic in each variable. This turns out to be equivalent to solving a set of problems restricted to a finite domain with periodic and quasi-periodic boundary conditions.

In [FKT], Feldman, Knörrer and Trubowitz show that a rich set of eigenvalue, eigenfunction pairs of Schrödinger's equation with periodic potential are perturbatively stable, which means that the difference between perturbed and unperturbed eigenvalues and eigenfunctions is asymptotically small, measured in inverse fractional powers of the eigenvalue; the error bound is given only for the first truncation of the expansions. They accomplish their result by disregarding small subsets of bad lattice points and their corresponding eigenvalue-eigenfunction pairs. In fact, this discarded set is defined by three conditions that involve geometric properties of the lattice. We will also discard a thin subset of bad lattice points. Our discarded set is defined by four conditions, two equivalent to theirs and two that are of a different nature but also depend only on geometric properties of the lattice points. 
In $[\mathrm{F}]$, Friedlander gives another proof for results similar to those in [FKT]. To do this, conditions equivalent to our first two are also imposed, plus an additional condition which uses information not known a priori, namely, the perturbed eigenvalues.

Both these papers are concerned only with the first truncation of the perturbation expansions, and both exhibit good estimates on the difference between perturbed and unperturbed eigenvalues. It should be noted that in both these papers, the bound for the difference between perturbed and unperturbed eigenfunctions is not an $L^{\infty}$ bound. Having an $L^{\infty}$ bound is necessary to use these estimates to solve the inverse nodal problem.

In $[\mathrm{KP}]$, Karpeshina exhibits the complete perturbation expansions for a rich set of eigenvalues and eigenprojectors for Schrödinger's equation with periodic potential with the additional assumption that the potential is a finite trigonometric polynomial in the spatial variables; she outlines how the procedure could be generalized for a smooth potential. Error estimates for truncations of these expansions are presented, and conditions defining subsets of bad eigenvalues are defined as well. The same pair of conditions common to all papers are represented here as well. The third condition in $[\mathrm{KP}]$ is similar in nature, but different, to ours.

In [KP2], Karpeshina presents the complete perturbation expansions for a rich set of eigenvalues and eigenprojectors for Schrödinger's equation with periodic potential. In this paper, assumptions on the smoothness of the potential are relaxed considerably. Estimates for errors of truncation are presented for a trace class norm, which, in fact, makes these estimates valid in $L^{\infty}$. The only drawback of the technique is that although the results are proved for a measure one subset of quasimomenta, a particular instance of quasimomenta for which the results are valid is not produced.

In what follows we present the statement of the key result in a setting that is not as general as possible, but makes ideas more accessible to the reader.

\section{MAIN RESULT}

We want to study the behavior of the eigenvalues and eigenfunctions satisfying

$$
-\Delta u+q u=\lambda u
$$

on the rectangular domain $R=[0, \pi / a] \times[0, \pi](a>1)$, with Dirichlet boundary conditions. Note that $q$ is in general not constant.

In order to better understand the effect of the $q u$, or restorative force term, we have to refer to the original equation from which this eigenvalue problem was derived, that is, the wave equation

$$
v_{t t}+g v=\rho^{2} \Delta v
$$

with Dirichlet boundary conditions and two prescribed initial conditions, where $\rho^{2}$ is the quotient of the constant tension over the constant density of the membrane. The term $g v$ is called restorative when $g \geq 0$ because it has the effect of wanting to return the system to a state of equilibrium or zero displacement. A way of thinking about the $g v$ term is to set $\rho^{2} \Delta v=0$ and study $v_{t t}+g v=0$. This is the equation governing the motion of a linear spring, where $g$ is a measure of the stiffness of springs attached to the membrane at each point in our domain. 
To obtain the eigenvalue problem we must consider solutions of the form $v=$ $e^{i \sigma t} u$, where $u$ is time independent. Plugging in and simplifying:

$$
-\sigma^{2} u+g u=\rho^{2} \Delta u \text {. }
$$

Slightly rearranging the terms we can see that $q=g / \rho^{2} \geq 0$ and $\lambda=\sigma^{2} / \rho^{2}$.

The eigenvalues and normalized eigenfunctions of (1) when $q \equiv 0$ are well known for rectangular domains:

$$
\begin{aligned}
& \lambda_{\alpha}=(a n)^{2}+m^{2}=|\alpha|^{2}, \\
& u_{\alpha}=\frac{2 \sqrt{a}}{\pi} \sin (\text { anx }) \sin (m y),
\end{aligned}
$$

where $\alpha \in L$ and our index set $L=\{(a n, m) \mid n, m=1,2,3, \ldots\}$ is an integer lattice. We will treat the case of $q \neq 0$ as a perturbation of the simpler, well-understood case $(q \equiv 0)$. So our new formulation for the problem will be

$$
-\Delta u(\epsilon)+\epsilon q u(\epsilon)=\lambda(\epsilon) u(\epsilon) .
$$

We use $\epsilon$ as a book-keeping parameter in the end set $\epsilon=1$, thus solving the original problem.

Theorem 1. Let $J=\left(1, a_{0}\right)$ be given. Define

$$
\begin{gathered}
V=\left\{a \in J \mid \text { there exist } 0<\delta<\epsilon_{0} / 6 \text { and } K>0\right. \text { such that for all } \\
\left.p, q>0:\left|a^{2}-\frac{p}{q}\right|>K / q^{2+\delta}\right\} .
\end{gathered}
$$

Let $q$ be such that

$$
\int_{R} q d x=0
$$

and

$$
|q|_{l}=\left\{\sum_{\alpha \in L}\left|\left(q, v_{\alpha}\right)\right|^{2}|\alpha|^{2 l}\right\}^{1 / 2}<\infty,
$$

with $l \geq 2$. Then there exists a finite, positive number $D_{1}$ and an exceptional set of lattice points $\breve{M}(a) \subset L(a)$ depending on a such that

a) meas $(J \backslash V)=0$ and $\breve{M}(a)$ has density 0 in $L(a)$ for all $a \in V$ in the sense that

$$
\lim _{r \rightarrow \infty} \frac{\# \breve{M}(a) \cap B(0, r)}{\# L(a) \cap B(0, r)}=0 .
$$

b) For every $\alpha \in L(a) \backslash\left(\breve{M}(a) \cup B\left(0, D_{1}\right)\right)$, there exists a circular contour $\Gamma_{\alpha}$ in the complex plane, centered at $\lambda_{\alpha}$, containing one and only one eigenvalue throughout the perturbation. Moreover, there is a unique eigenvalue of the variable coefficient problem (1), which we denote by $\lambda_{\alpha q}$, satisfying

$$
\left|\lambda_{\alpha q}-\lambda_{\alpha}\right| \leq(2+30 \sqrt{a}|q|)|q|_{l}^{2}|\alpha|^{-1 / 2+2 \delta} .
$$

This is the bound for the error made by the 1st truncation of the perturbation expansion for the eigenvalue. In general, the perturbation expansion for the eigenvalue 
is given by

$$
\begin{aligned}
\lambda_{\alpha q}= & \lambda_{\alpha}+\sum_{n=0}^{\infty}(-1)^{n} \sum_{\beta_{0} \in L} \cdots \sum_{\beta_{n} \in L}\left\{\frac{1}{2 \pi i} \int_{\Gamma_{\alpha}} \frac{\left(\lambda_{\beta_{0}}-\lambda_{\alpha}\right)\left(u_{\beta_{0}}, q u_{\beta_{1}}\right)}{\left(\lambda_{\beta_{0}}-\xi\right)^{2}}\right. \\
& \times \frac{\left(u_{\beta_{1}}, q u_{\beta_{2}}\right)}{\lambda_{\beta_{1}}-\xi} \frac{\left(u_{\beta_{n}}, q u_{\beta_{0}}\right)}{\lambda_{\beta_{n}}-\xi} d \xi \\
& \left.-\frac{1}{2 \pi i} \int_{\Gamma_{\alpha}} \frac{\left(u_{\beta_{n}}, q u_{\beta_{0}}\right)}{\lambda_{\beta_{0}}-\xi} \frac{\left(u_{\beta_{0}}, q u_{\beta_{1}}\right)}{\lambda_{\beta_{1}}-\xi} \cdots \frac{\left(u_{\beta_{n-1}}, q u_{\beta_{n}}\right)}{\lambda_{\beta_{n}}-\xi} d \xi\right\},
\end{aligned}
$$

and $a$ bound for the error made by the mth truncation is

$$
\sqrt{a}|q| 12(m+1)|q|_{l}^{m-1}|\alpha|^{(-1 / 4+\delta)(m-1)} \text {. }
$$

A suitable multiple of the corresponding eigenfunction satisfies

$$
\left\|u_{\alpha q}-u_{\alpha}\right\|_{L^{\infty}} \leq 3 \sqrt{a}|q|_{l}^{2}|\alpha|^{-1 / 2+2 \delta}
$$

This is a bound for the error made by the 1st truncation of the perturbation expansion for the eigenfunction. In general, the perturbation expansion of the eigenfunction is given by

$$
\begin{aligned}
u_{\alpha q}= & u_{\alpha}+\sum_{n=1}^{\infty}(-1)^{n+1} \sum_{\beta_{0} \in L} \cdots \sum_{\beta_{n-1} \in L} \frac{1}{2 \pi i} \int_{\Gamma_{\alpha}} \frac{\left(u_{\beta_{0}}, q u_{\beta_{1}}\right)}{\lambda_{\beta_{0}}-\xi} \times \cdots \\
& \times \frac{\left(u_{\beta_{n-2}}, q u_{\beta_{n-1}}\right)}{\lambda_{\beta_{n-2}}-\xi} \frac{\left(u_{\beta_{n-1}}, q u_{\alpha}\right)}{\lambda_{\beta_{n-1}}-\xi} \frac{1}{\lambda_{\alpha}-\xi} u_{\beta_{0}} d \xi
\end{aligned}
$$

and a bound for the $L^{\infty}$ error made by the mth truncation is

$$
2 \sqrt{a}|q|_{l}^{m}|\alpha|^{(-1 / 4+\delta) m}
$$

where $0<\delta<1 / 10$ can be made arbitrarily small.

Remark. The reader will observe that the bound given for the error made by the 1st truncation for both the eigenvalue and eigenfunction is stronger than that implied by the error for the 1st truncation of the series expansion. To obtain the better bound we more carefully bound the $n=1$ and $n=2$ terms of the corresponding series expansion.

Because our main contribution involves the definition of the exceptional subset $\breve{M}(a) \subset L(a)$, we present its defining elements in what follows:

$$
\begin{aligned}
& M_{10}(a)=\left\{\alpha \in L \mid \text { there exists } \beta \in L, \beta \neq \alpha \text {, and }\left.|| \alpha\right|^{2}-\left.|\beta|^{2}\left|<C_{0}\right| \alpha\right|^{-\epsilon_{0}}\right\}, \\
& M_{11}(a)=\left\{\alpha \in L \mid \text { there exists } \beta \in L, \beta \neq \alpha \text {, and }|\alpha-\beta|<C_{1}|\alpha|^{\epsilon_{1}},\right. \\
& \left.\left|\lambda_{\alpha 0}-\lambda_{\beta 0}\right|<C_{2}|\alpha|^{1-\epsilon_{2}}\right\} \\
& M_{12}(a)=\left\{\alpha=(a n, m) \in L \mid \text { we have that } m<\left(\delta C_{1}\right)^{1 /(1-p)}(a n)^{p}\right. \text { or } \\
& \text { an } \left.<\left(\delta C_{1}\right)^{1 /(1-p)} m^{p}\right\} \\
& M_{13}(a)=\left\{\alpha \in L \backslash M_{10} \quad \mid \exists \beta, \gamma \in L, \beta, \gamma \neq \alpha\right. \\
& 0<|\beta-\gamma|<C_{1}|\alpha|^{\epsilon_{1}} \\
& \left.\left|\lambda_{\beta 0}-\lambda_{\alpha 0}\right|\left|\lambda_{\gamma 0}-\lambda_{\alpha 0}\right|<C_{3}|\alpha|^{\epsilon_{3}}\right\} \text {, }
\end{aligned}
$$


with the following restrictions on the parameters:

$$
\begin{gathered}
0<\epsilon_{0}<1 / 2, \quad 0<\epsilon_{1}<\epsilon_{2}<1 / 2, \\
0<p<1, \quad l \geq 2, \\
l>\max \left\{\left(1-\epsilon_{2}\right) / p, 1+\left(3 \epsilon_{0}\right) /\left(2 \epsilon_{1}\right), 1+\left(\epsilon_{3}+\epsilon_{0}\right) / \epsilon_{1}\right\}, \\
0<\epsilon_{0}<\left(\epsilon_{2}-\epsilon_{1}\right) / 2, \quad 0<\epsilon_{3}<\epsilon_{2}-\epsilon_{1}-2 \epsilon_{0}, \\
C_{0}>C_{3}, \\
2\left(C_{3} / C_{0}\right)\left(1 / \sqrt{1-C_{3} / C_{0}}\right)^{\epsilon_{3}+\epsilon_{0}}<C_{2} .
\end{gathered}
$$

Remark. $\breve{M}(a)=M_{10}(a) \cup M_{11}(a) \cup M_{12}(a) \cup M_{13}(a)$. The subsets $M_{10}(a), M_{11}(a)$ and $M_{12}(a)$ have been previously defined and used in the literature; the set $M_{13}(a)$ is our contribution and is essential for our analysis.

\section{REFERENCES}

[MP] Joyce R. McLaughlin and Arturo Portnoy, Perturbing a rectangular membrane with a restorative force: effects on eigenvalues and eigenfunctions, to appear in Communications in Partial Differential Equations.

[HM] Ole H. Hald and Joyce R. McLaughlin, Inverse nodal problems: finding the potential from nodal lines, Memoirs of the American Mathematical Society, January 1996, Volume 119, Number 572. MR 97d:35240

$[\mathrm{MH}]$ Joyce R. McLaughlin and Ole H. Hald, A formula for finding a potential from nodal lines, Bulletin (new series) of the American Mathematical Society (1995), Volume 32, Number 2, pp. 241. MR 95g:35213

[FKT] J. Feldman, H. Knörrer and E. Trubowitz, The perturbatively stable spectrum of a periodic Schrödinger operator, Inventiones Matematicae, Vol. 100 (1990), pp. 259-300. MR 91 m:35167

[F] L. Friedlander, On the spectrum of the periodic problem for the Schrödinger operator, Comm. P.D.E., Vol. 15 (1990), pp. 1631-1647. MR 92i:35092a

[KP] Yu. E. Karpeshina, Analytic perturbation theory for a periodic potential, Math. USSR Izvestiya, Vol. 34 (1990), No.1, pp. 43-64. MR 91f:47065

[KP2] Yu. E. Karpeshina, Geometrical background for the perturbation theory of the polyharmonic operator with periodic potentials, International Seminar on Geometrical Aspects of Quantum Theory, Topological Phases in Quantum Theory, World Scientific, 1988, pp. 251-276. MR 93c: 81044

[K] Tosio Kato, Perturbation theory for linear operators, New York: Springer-Verlag, 2nd Ed., 1976. MR 53:11389

[S] Wolfgang M. Schmidt, Diophantine approximations and Diophantine equations, New York: Springer Verlag, 1991. MR 94f:11059

[TL] Angus E. Taylor and David C. Lay, Introduction to functional analysis, New York: Wiley, 1980. MR 81b:46001

Rensselaer Polytechnic Institute, Troy, Ny 12180

E-mail address: mclauj@rpi.edu

Rensselaer Polytechnic Institute, Troy, NY 12180

E-mail address: portna@rpi.edu 